\title{
Essais
}

Revue interdisciplinaire d'Humanités

$10 \mid 2016$

Faire-valoir et seconds couteaux

\section{Neverwhere : les égarés de la narration}

\section{Aurélien Royer}

\section{OpenEdition}

Journals

Édition électronique

URL : http://journals.openedition.org/essais/3917

DOI : $10.4000 /$ essais.3917

ISSN : 2276-0970

\section{Éditeur}

École doctorale Montaigne Humanités

\section{Édition imprimée}

Date de publication : 15 septembre 2016

Pagination : 97-106

ISBN : 978-2-9544269-9-0

ISSN : 2417-4211

Référence électronique

Aurélien Royer, "Neverwhere : les égarés de la narration », Essais [En ligne], 10 | 2016, mis en ligne le 15 octobre 2020, consulté le 21 octobre 2020. URL : http://journals.openedition.org/essais/3917 ; DOI : https://doi.org/10.4000/essais.3917 


\section{Newerwhere: les égarés de la narration}

Aurélien Royer

\section{Introduction}

Neverwhere est un roman fantastique écrit par Neil Gaiman en 1996, à la suite de l'écriture et de la diffusion sur la BBC de la série du même nom. Confronté aux coupes dans son scénario original, coupes rendues nécessaires par le format télévisuel et les contraintes de production, N. Gaiman raconte dans son introduction à l'édition 2005 du roman, qu'il n'a surmonté cela qu'en disant par devers lui qu'il remettrait le tout dans le roman ("I'll put it back in the novel ", I) ${ }^{1}$. Dès l'origine, nous constatons une frustration (véridique ou feinte) à ne pouvoir tout dire, tout montrer, tout raconter. Dans cette optique, la forme romanesque de Neverwhere serait censément la plus complète. Or, et c'est ce à quoi nous allons nous intéresser au cours de notre examen, le texte apparaît étonnamment troué pour quelque chose de prétendument entier. Cela est d'autant plus flagrant que le récit prend souvent la peine de sortir de ses rails pour éclairer quelque chose qui, sans cela, serait resté dans l'obscurité. Des informations manquent, d'autres surgissent, et c'est à nous, lecteurs, qu'il revient de recoller les morceaux. Ce jeu de lumières et d'ombres est rendu possible avant tout par la focalisation du récit. Cette dernière, plutôt que de suivre constamment un personnage principal, est éclatée. Bien sûr, elle s'intéresse surtout à un personnage précis, un héros par défaut, dirons-nous, mais aussi à la galerie de personnages secondaires qui l'entoure, que les dénominations de faire-valoir ou de seconds couteaux ne saisissent qu'improprement. De sorte que finalement le statut des personnages s'estompe jusqu'à disparaitre. Il n'y a pas de héros de plein droit dans Neverwhere et, par là même, il n'y a pas de personnage proprement secondaire. Chacun devient l'adjuvant ou

1 Toutes les références à Neverwhere seront, par la suite, matérialisées par un numéro de page entre parenthèses. Sauf indication contraire, toutes les citations sont issues de : Gaiman, Neil, Neverwhere, Londres : Review, 2005. Enfin, précisons que les numéros de page de l'introduction sont les miens, l'édition utilisée ne les numérotant pas. 
l'opposant de l'autre, changeant plusieurs fois de rôle dans le récit, sans jamais atteindre de stabilisation dans une fonction ou une autre. Les personnages du roman semblent ainsi perdus, égarés dans une narration qui les déplace à loisir. C'est ce mouvement que je souhaiterais explorer dans ce texte, en montrant en quoi chaque personnage du récit est un héros qui s'est trompé d'histoire, en quoi chaque personnage du récit est un faire-valoir sporadiquement éclairé par la narration.

Pour ce faire, il convient tout d'abord de proposer un aperçu de l'œuvre. Ceci sert de tremplin à l'analyse des schémas actantiels ${ }^{2}$ du roman, analyse qui montre combien les fonctions des personnages sont opaques. L'enjeu étant de mettre au jour le statut duel du roman dont est issue cette perte de repères fonctionnels qui frappe le récit.

\section{Méandres}

Comme précisé en introduction, Neverwhere fut d'abord le scénario d'une série télévisuelle britannique. N. Gaiman a réécrit et étendu ce scénario sous forme de roman, publié par la BBC en 1996, la semaine de diffusion du troisième épisode de la série. Un éditeur américain a ensuite offert à l'auteur de proposer le roman aux États-Unis et N. Gaiman en a profité pour rédiger une nouvelle mouture du texte. Cette version parut en 1998. Finalement, une édition définitive (intitulée "The Author's Preferred Text") vit le jour en 2005 chez Review et c'est sur celle-ci que nous nous appuierons. Pour cette édition, $\mathrm{N}$. Gaiman réunit les versions antérieures, supprimant et ajoutant quelques milliers de mots (" a few thousand words ", II). Voici, en quelques mots, la génétique du récit.

Malgré cette genèse complexe, le roman présente une fabula d'apparence simple. L'œuvre nous fait suivre les péripéties du personnage focalisateur principal nommé Richard Mayhew, analyste financier écossais immigré à Londres, vivant une existence sans intérêt au début du roman. Sa rencontre avec un second personnage va bouleverser tout ceci et, dans la tradition populaire des romans de formation, le voici plongé dans une série d'aventures qui lui permettra, à terme, de se connaître lui-même. En sus de l'introduction, le péritexte du récit se compose d'un prologue placé, naturellement, au début et, en annexe, d'un prologue tout à fait différent (" an altogether different prologue ", 377), que nous évoquerons plus tard. Pour l'instant, c'est le prologue de plein droit qui doit retenir notre attention. Nous y voyons Richard Mayhew fêter son départ d'Écosse dans une ambiance un peu amère, au milieu de ses anciens camarades de travail.

2 Pour ce faire, nous nous servirons du modèle établi par Greimas, Algirdas Julien dans Sémantique Structurale, Paris : PUF, 1986 (2007), p. 180. 
Ce point de départ mérite examen. En tant que prologue rédigé par l'auteur lui-même, il participe de la catégorie des préfaces originales qui " [ont] pour fonction cardinale d'assurer au texte une bonne lecture " ${ }^{3}$. De sorte que, par sa visée programmatique, le prologue «fournit le mode d'emploi du livre ${ }^{4}$ : il dirige l'attention du lecteur, il oriente la lecture. Ainsi, en étant focalisé sur Richard, le prologue en fait le personnage focalisateur mais aussi le personnage principal. En nous présentant cette première aventure - où, très ironiquement, Richard se fait lire son avenir - le prologue établit dans l'horizon d'attente du lecteur le personnage de Richard comme héros. Or, le premier paragraphe du texte opère un changement radical puisqu'en place de Richard, c'est un pronom personnel sujet féminin (SHE) qui apparaît, anonyme et fuyant quelque chose. Aussitôt après, la focalisation présente un nouveau déplacement en mettant en lumière Ms. Croup et Vandemar, poursuivants du personnage précédent. C'est seulement trois pages plus loin que nous retrouvons Richard, de nouveau focalisateur. Cette hésitation du texte va se poursuivre jusqu'à la fin du premier chapitre où finalement elle sera résolue, rappelant le procédé cinématographique du montage alterné (on pense notamment au final du Parrain, par Francis F. Coppola).

Le texte hésite ainsi dès l'abord sur sa composition. Il n'y a pas d'exposition proprement dite d'un personnage principal comme cela peut-être le cas dans la plupart des romans populaires (voyez plutôt l'introduction des personnages chez T. Pratchett, ou encore les premières pages généalogiques de The Lord of the Rings). Au contraire, au travers de ce montage alterné, le récit échappe à toute tentative de stabilisation.

Notons encore que la résolution du parallélisme permet au lecteur d'envisager l'hypothèse d'un personnage principal double mais cette idée vole assez vite en éclat. En effet, très rapidement, à la fin du deuxième chapitre, Door, le personnage féminin, qui avait rejoint Richard, le quitte. À partir de ce moment, le récit, bien que demeurant principalement focalisé sur Richard alterne de nouveau avec des scènes évoquées via Door, ou Croup et Vandemar ou d'autres personnages encore. De sorte que, pendant quelques pages, des personnages qui semblent secondaires servent de héros de plein droit au récit. C'est le cas par exemple de Croup et Vandemar à l'occasion du second prologue, où le lecteur les aperçoit regardant brûler un monastère toscan auquel ils ont mis le feu. Les mini-séquences de récit de ce type sont légion dans Neverwhere et elles brouillent activement les repérages fonctionnels des personnages.

Mais revenons à notre récit. En fin de premier chapitre, Richard sauve Door de ses poursuivants. Elle va alors lui demander d'aller chercher quelqu'un pour l'aider. Richard se retrouve alors à servir d'adjuvant pour un person-

3 Genette, Gérard, Seuils, Paris : Seuil (coll. Points Essais), 1987 (2002), p. 200. Sauf indication contraire, toutes les italiques des citations sont de leurs auteurs.

4 Novalis, Encyclopédie, in Genette, Gérard, Seuils, op. cit., p. 212. 
nage principal effacé pour aller chercher un second adjuvant, tout en restant l'objet de la focalisation du récit. On perçoit ainsi combien les fonctions sont poreuses. C'est ainsi que Richard ramène à Door le Marquis de Carabas. Ces deux derniers quittent Richard. Comme de juste, l'histoire ne s'arrête pas ici : Door, le Marquis ainsi que Croup et Vandemar sont issus d'un monde, London Below, invisible aux yeux de ceux d'en dessus. Richard a été contaminé par ces personnages et se retrouve précipité dans London Below contre son gré.

Cette chute est l'occasion de reprendre le montage alterné, faisant suivre au lecteur tantôt l'enquête du Marquis et de Door pour retrouver les assassins de la famille de la jeune fille, tantôt les mésaventures de Richard, tantôt la marche différée de Croup et Vandemar. Un groupe se constitue finalement lorsque Richard retrouve le Marquis et Door alors qu'ils recrutent un garde du corps. Celui-ci sert d'adjuvant à Door et se nomme Hunter. Nous étudierons ce nom, programmatique comme beaucoup d'autres, plus bas. Pendant un temps, l'hésitation narrative s'estompe à quelques regards près chez Croup et Vandemar. La narration reste ainsi unifiée jusqu'au départ du Marquis pour un acte héroïque où il trouve une mort provisoire. En parallèle, Hunter trahit Door par intérêt personnel mais échoue dans sa quête et meurt. Ensuite, Richard, Door et le Marquis ressuscité affrontent le responsable de la mort de la famille de Door, l'ange Islington. Une fois celui-ci et Croup et Vandemar vaincus, Richard récupère sa vie d'avant. Bien entendu après toutes ses aventures, il ne s'en satisfait pas et retourne dans London Below, dont il est à présent un membre tutélaire, "the warrior" $(319,347)$.

Par volonté d'efficacité, j'ai limité ce résumé aux événements majeurs du récit et ainsi tu l'existence de personnages moins importants. Pourtant, tout incidentels qu'ils sont, il est nécessaire d'au moins citer leurs noms pour référence, car ceux-ci les inscrivent dans un espace extradiégétique et donc participent à l'indécision du statut des personnages. Nommons alors Old Bailey, le comte (The Earl), les Black Friars ou encore Lady Serpentine. Nous nous intéresserons à ces noms un peu plus loin.

\section{Cadres}

Dans un premier temps, parce que nous nous sommes surtout intéressés à Richard, notre résumé nous invite à considérer le récit comme un roman de formation. La focalisation principale du récit sur Richard, son émancipation finale, la découverte de son nom, son adoubement et son retour à London Below en font un personnage arrivé, qui contraste fortement avec le personnage effacé, manipulé et mièvre présenté en début du roman. Ce contraste est rendu possible par le dernier chapitre qui fait agir Richard dans le monde du Londres d'en haut et le montre lassé par celui-ci. 
The old Richard, the one who had lived in what was now the Buchanans' home, would have crumbled at this point, apologised for being a nuisance, and gone away. Instead, Richard said "Really"... (358-359)

Ce chapitre de conclusion permet de cadrer le récit sur Richard. Cependant, ce cadrage large de roman de formation n'arrive pas à subsumer l'ensemble des événements du récit, ne serait-ce que du fait que le roman ne commence pas sur Richard mais sur Door. Ainsi, le récit de la formation de Richard serait incidentel, secondaire. Richard n'est pas le personnage qui déclenche les aventures et encore moins celui qui les résout, à deux exceptions notables près. Il est celui qui subit, toujours de manière piteuse. Ainsi, par exemple, l'épisode $\mathrm{du}$ "Mind the gap thing" (141-142).

Ceci s'expliquerait par le fait que Richard glisse dans la mauvaise histoire au moment précis où il sauve Door agonisante sur le trottoir. Jusqu’à cet instant, il suivait un chemin narratif banal, classique, qui s'impose une dernière fois à lui à la fin du récit quand il voit son avenir se dérouler devant ses yeux :

And it came to him then [...]: the rest of his life. He would go home tonight with the girl from Computer Services, and they would make gentle love, and tomorrow, it being Saturday, they would spend the morning in bed. [...] In a year, or a little less, he would marry the girl from Computer Services, and get another promotion, and they would have two children, a boy and a girl, and they would move out to the suburbs... (365)

Ce récit, que Richard refuse en fin de roman, est pourtant celui qu'il pourchasse précisément tout le long de l'histoire : "I'm trying to get to the real London, and my old life " (227). Ajoutons encore pour renforcer l'idée d'égarement du personnage que, avant sa chute dans London Below, Richard était mené de manière caricaturale par sa future femme, qui le malléait selon ses désirs : "And Jessica saw in Richard an enormous amount of potential which, harnessed by the right woman, who would have made him the perfect matrimonial accessory. " (12). Ainsi, du début jusqu'à la fin, Richard est un personnage littéralement égaré dans les mauvaises histoires.

Non seulement Richard peine à déchiffrer le récit de London Below (274) mais en plus il interrompt les récits des autres, les parasite, parce qu'il est le focalisateur ${ }^{5}$ principal. Il agit comme un trou noir narratif. Chaque récit où il intervient subit une défocalisation qui prive le personnage principal de la lumière qu'il attendrait. Chaque fois qu'un des personnages du récit se retrouve accompagné par Richard, celui-ci devient automatiquement le focalisateur de la narration, quand bien même il ne s'agit pas de son récit et que la narration de ce dernier était précédemment focalisé sur l'autre personnage. Richard fonctionne alors comme un personnage transversal, un faire-valoir

5 Nous reprenons ici les termes définit par Genette, Gérard, Figures III, Paris : Seuil (coll. Poétique), 1972, p. 200 et sq. 
qui permet au récit de mettre en lumière tout ce qui arrive autour de lui. Personnage transversal car il participe de toutes les séquences du récit. Hunter déclare ainsi : "I saved his life three times today ", 123) pour justifier de ses qualités de garde du corps. Plus tard, il passera la troisième épreuve de la quête que Door doit accomplir pour obtenir une clef. Plus tard encore, il accompagnera le Marquis pour aller sauver Door.

Il serait alors tentant de le classer définitivement dans les personnages secondaires, adjuvant maladroit de tous les autres, mais deux faits s'y opposent. D'abord sa victoire sur la Bête de London Below, où il devient un héros de plein droit (et un membre du monde d'en bas, ne serait-ce que par intertextualité). Ensuite, comme il constitue le focalisateur principal, il permet au narrateur de déployer les merveilles du monde sans lasser et avec la fraîcheur de la nouveauté, tout en présentant un visage auquel le lecteur peut s'identifier aisément. Richard est donc double. À la fois personnage principal répondant à des besoins narratifs précis et personnage secondaire dans les schémas actantiels du récit.

En effet, Neverwhere compte presque autant de schémas actantiels que de personnages. Pour comprendre cela, appuyons-nous d'abord sur le résumé du récit aimablement proposé par Richard dans une question à Hunter : "So what are you after? [...] Personally I'm trying to get to real London and my old life. Door wants to find out who killed her family. What are you after?" (227). La réponse de Hunter est amusante car elle raconte son histoire passée (l'inventaire des bêtes qu'elle a tuées) avant de déclarer qu'elle tuera celle de Londres ou mourra en essayant. Amusante car elle nous permet de situer Hunter sur une ligne narrative déviante par rapport à celle de Door ou de Richard. Hunter, comme son nom l'indique, chasse. Sa coopération avec Door ne peut apparaître qu'incidente.

La question de Richard permet d'identifier la quête de Door et c'est cette quête qui réunit toutes les autres. Le schéma actantiel qui correspond à la séquence de Door est le suivant :

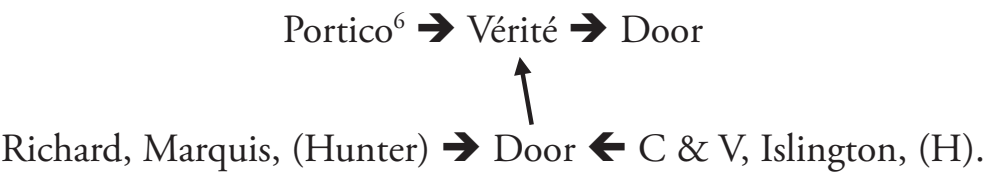

On s'aperçoit alors qu'il réunit tous les personnages autour de Door qui n'est qu'un adjuvant dans le récit de formation de Richard alors même que le texte s'ouvre sur son aventure. D’autre part, Door est révérée par les personnages de London Below (elle est Lady Door), elle n'est inféodée à personne et possède un pouvoir magique (elle ouvre les choses). Tout ceci s'ajoute au schéma actantiel pour faire d'elle un héros ${ }^{7}$ prototypique des romans populaires, qui va surmon-

6 Père de Door, dont le testament enregistré (et truqué) lance Door sur le chemin de son récit.

7 Comme il s'agit ici du rôle prototypique de héros, hors de toute spécificité, nous conservons le 
ter les obstacles pour arriver à ses fins. Sauf qu'elle ne sert pas d'héroïne au roman : le personnage est un faire-valoir brillant de Richard, un contrepoint qui dévoile toute l'ignorance du précédent. Ceci nous permet d'explorer un peu plus l'idée d'égarement. Door ne s'est pas trompé d'histoire. À l'inverse de Richard, elle sait où elle trouve, d'où elle est partie et où elle va.

Cependant, la narration choisit délibérément de l'exclure (voir le prologue et le dernier chapitre, tous les deux centrés sur Richard) pour raconter une autre histoire. Le personnage de Door est un faire-valoir avant tout dans la mesure où la narration le décide, de la même façon que Richard, faire-valoir archétypique devient le personnage principal par les manipulations du narrateur. Ainsi, même si le récit de Door unit tous les autres, y compris celui, cadre, de la formation de Richard, il n'en reste pas moins secondaire. C'est, cette fois-ci, le narrateur qui se trompe de lumière, pourrait-on dire.

Nous obtenons donc un héros d'un récit principal travesti en adjuvant d'un récit accessoire et structurant ; et un faire-valoir du récit principal maquillé en personnage principal du récit secondaire, ce qui annonce la complexité des autres personnages. Door et Richard présentent en effet l'avantage de se partager l'affiche. Tous les deux sont les personnages principaux des récits qui se télescopent. Même si l'hypothèse du double héros ne tient pas ${ }^{8}$, ils n'en restent pas moins tous les deux à l'avant-scène. Les autres personnages révèlent des structures tout aussi plastiques.

C'est principalement le cas pour le Marquis de Carabas dont le nom indique une origine clairement extradiégétique. Le marquis de Carabas est la fausse identité que revêt le fils de meunier sous l'instigation du Chat Botté dans le conte de Perrault ${ }^{9}$. Ici, le rôle est inversé : le Marquis est un flamboyant adjuvant et, comme son nom l'indique, un mensonge. Le marquis énonce lui-même s'être construit comme une vaste blague (" a grand joke ", 239). Au-delà du nom, le Marquis témoigne de sa portée extradiégétique dans ses aventures au sein même du récit. De fait, le Marquis quitte le groupe principal à un moment du récit et devient le focalisateur pour un temps, se faisant ainsi le " héros de sa propre séquence ${ }^{10}$. Et c'est effectivement un acte héroïque auquel il se livre puisqu'il va défier dans leur tanière les incarnations de la mort que sont Croup et Vandemar. Il paie cette hubris de sa vie. En mourant, il échappe littéralement au récit, pour y revenir plus tard, ressuscité, juste à temps pour sauver Richard. En quelques pages, le Marquis couvre ainsi toutes les fonctions actantielles du schéma et se permet de vagabonder hors du monde narratif.

masculin, censément neutre en français. À l’inverse, deux lignes plus bas, comme nous évoquons le rôle particulier que Door aurait pu endosser dans ce récit précis, nous utilisons le féminin.

8 Ne serait-ce que par la présence des autres personnages à la fois secondaires et héros : le Marquis et Hunter.

9 Perrault, Charles, Contes, Paris : Garnier Flammarion 1991 (ed. Soriano, Marc), p. 267.

10 Barthes, "Introduction à l'analyse structurale des récits " (1966), in Barthes, Kayser, Booth, Hamon, Poétique du récit, Paris : Seuil (coll. Points essais), 1977, p. 34. 
Hunter subit une transformation équivalente et encore plus rapide puisqu'en l'espace de quelques paragraphes, elle passe d'adjuvant à opposant, puis à personnage principal de l'affrontement avec la bête, pour redevenir adjuvant et finir cadavre. Ces transformations successives nous permettent de comprendre qu'Hunter participe d'un schéma actantiel qui lui est propre. Elle suit un récit particulier : sa chasse. Dans cet ordre d'idée, son appartenance au schéma de Door est incidentelle.

Les personnages les plus extérieurs au récit montrent de leur côté une nature profondément extradiégétique. Il s'agit des personnages que j’ai nommés plus haut et dont les noms font systématiquement référence à la réalité géographique de Londres : Serpentine, Old Bailey, Islington, les Black Friars ou encore le Comte (Earl). En étant directement liés à la géographie, ils s'inscrivent non pas dans le récit, transitoire, du roman, mais s'insèrent dans un récit géographique, démesurément plus ancien que le récit du roman, qui le sous-tend, l'appuie, le nourrit et lui fournit une forme de stabilisation profonde qui lui est refusée partout ailleurs.

\section{Éclatements}

Avant ce soubassement géographique, il existe deux récits. En effet, deux Londres cohabitent, nous l'avons vu précédemment : en bas et en haut. Cette différence nous renseigne sur le statut des personnages car leur déplacement sur cet axe vertical est en fait un déplacement narratif. Neverwhere est marqué par une dichotomie narrative puissante. Les noms des personnages de London Below nous le montrent dans un premier temps : soit qu'ils s'inscrivent dans un texte extradiégétique (les toponymes), soit qu'ils témoignent de leur fonction (Hunter, Door), ils prouvent que London Below est fondée sur un autre langage que le Londres d'en haut, ainsi que l'exprime Richard : " He had gone beyond the world of metaphore and simile, into the place of things that are. " (310-311). Ce déplacement est d'autant plus acté que Richard perd son nom pour gagner une fonction : "warrior». À l'inverse, le Londres d'en haut ne marque pas une telle opérabilité des noms, à tel point que Gary, le collègue de Richard, devient très vite "Gary-from-work " (tirets inclus) et que la potentielle compagne de Richard demeure " the girl from Computer Services». Quand les noms de London Below témoignent d'une essentialisation de leur possesseur, ceux du Londres d'en haut sont au contraire les marqueurs d'une réification.

Il faut donc considérer Neverwhere comme un triple empilement narratif qui viserait à appréhender la réalité de manière de plus en plus immédiate. Le monde d'en haut, celui des métaphores et des comparaisons, serait un échec dans cette entreprise, attendu que les mots ne rendent pas le réel tel qu'il est. Le monde d'en bas chercherait, par ses dénominations fonctionnelles (un personnage se nommant Hammersmith est bien évidemment forgeron par 
exemple), à réduire au mieux la distance entre le signe et le signifié, distance qui serait abolie dans le troisième monde narratif, le monde souterrain des personnages géographiques et extradiégétiques.

C'est au titre de cet éclatement narratif qu'il est possible de parler d'égarés narratifs lorsqu'on regarde les personnages de Neverwhere. Richard est égaré dans le monde d'en haut puis dans celui d'en bas et cherche, dans les deux, une fonction narrative à remplir, il cherche à devenir réel et le devient en retournant dans London Below. Door s'égare en haut puis dans l'histoire de Richard et celui-ci est incapable de comprendre sa fonction, faute de langage commun. Hunter, dans sa traque, reste collée à sa fonction narrative mais ne s’intègre jamais au récit. Enfin, le Marquis échappe à l'immédiateté du langage que Neverwhere cherche à instaurer dans London Below, tant et si bien qu'il échappe au récit lui-même et ne peut rapporter son voyage que dans des termes vagues. Pourtant, malgré cet égarement permanent, le Marquis reste fidèle à sa fonction d'adjuvant. Il serait possible, en exagérant un peu, de supposer que précisément parce qu'il est libre et volage, il ne peut remplir qu'une seule fonction, la sienne. Dans cette optique, le Marquis de Carabas, personnage mensonge, serait le seul personnage vrai du roman.

\section{Conclusion}

La multiplicité des mondes narratifs du roman, l'éclatement des fonctions actantielles et leur distribution aléatoire, la focalisation qui voyage, les personnages qui ne se trouvent pas de rôle définitif et définitoires, l'absence de récits totalisants, la série de récits coïncidant par hasard narratif, tous ces éléments font de Neverwhere un texte complexe. Dans Neverwhere, "La fonction narrative [a perdu] ses foncteurs, [son] grand héros, [ses] grands périls, [ses] grands périples et [son] grand but ${ }^{11}$. Il n'y a pas à proprement parler de faire-valoir ou de seconds couteaux ou de personnage principal dans le roman. En lieu et place de cela, nous voyons un tourbillon de personnages qui occupent successivement tous les rôles. Seule la focalisation permettrait de forcer le roman dans les cadres narratifs habituels. Neverwhere apparaît comme un roman décentré et postmoderne ${ }^{12}$.

Surtout, Neverwhere se positionne en esquive permanente, refusant toute stabilisation au point tel qu'à la fin du roman, alors que Richard retourne à London Below pour devenir une fonction, pour se stabiliser enfin et trouver sa réalité, nous ne voyons rien :

11 Lyotard, Jean-François, La condition postmoderne, Paris : Minuit, 1979, p. 7-8.

12 Nous avons cherché ici à le montrer au niveau narratif. Pour une discussion sur la postmodernité de Neverwhere au niveau symbolique, voir Romero-Jódar, Andrés, « Paradisiacal Hells. Subversions of the mythical canon in Neil Gaiman's Neverwhere ", in Cuadernos de Investigación Filológica 31-32 (2005-2006), p. 163-195. 
And they walked away together through the hole in the wall back into the darkness, leaving nothing behind them; not even the doorway. (372)

\author{
Aurélien Royer \\ EA 4196 CLIMAS \\ Université Bordeaux Montaigne \\ Aurelien.Royer@u-bordeaux-montaigne.fr
}

\begin{abstract}
This article focuses on the characters of the novel Neverwhere written by Neil Gaiman and discusses the roles they play in the novel. It tackles their mutability and their lack of stability through a careful analysis of the narrative patterns dictating their actions. Its leading statement is that, despite being presented as sidekicks and underlings, all the characters in the novel are in fact heroes in their own rights, albeit of different stories.
\end{abstract}

\title{
Keywords
}

Sidekicks, Neil Gaiman, Neverwhere, fantasy literature.

\section{Résumé}

Cet article propose une analyse des schémas actantiels qui régissent les différents personnages du roman Neverwhere par Neil Gaiman. À travers une exploration rigoureuse des rôles successivement endossés par les personnages, il cherche à démontrer la porosité des fonctions narratives classiques. Au terme de cette démarche, on s'aperçoit que le récit met en acte des personnages mouvants, chacun héros, de leur histoire ou d'une autre.

\section{Mots-clés}

Faire-valoir, Neil Gaiman, Neverwhere, littérature fantastique. 